\title{
Dual-Position-Controller Design for the Linear-Motor-Driven Motion System*
}

\author{
Yuan-Yong HUANG**, Rong-Hwang HORNG**, Yi-Ti SHIH*** \\ and An-Chen LEE** \\ ** Department of Mechanical Engineering, National Chiao-Tung University, \\ 1001 Ta-Hsueh Road, Hsinchu City, TAIWAN, R.O.C. \\ E-mail: aclee@mail.nctu.edu.tw \\ *** ICP DAS CO., LTD
}

111, Kuangfu N. Rd., Hukou Shiang, Hsinchu, TAIWAN, R.O.C.

\begin{abstract}
This work develops a dual-controller composed of a macroscopic controller (MAC) and a microscopic controller (MIC) for improving motion precision of a linear-motor-driven motion system. Based on the macroscopic model in which Coulomb friction model is considered, the MAC is designed. In the presliding region however, the MIC design is based on the lineralized microscopic model. Furthermore, a switching algorithm is developed for bumpless transfer in shifting control action between two controllers. Thus, when the table of motion stage moves to the desired position, the control action can be smoothly switched from the MAC to the MIC. The whole system with the proposed dual-controller has the advantage that it serves as a long stroke (coarse stage) and a short stroke (fine stage) to achieve high precision motion control. The experimental results reveal that it totally takes 2.59 seconds to reach the $1000 \mu m$ target position with the accuracy of one BLU (basic length unit; sensor resolution), 20nm; the result has over $29 \%$ improvement when compared with the result using single MAC. In addition, good nanometer-scale tracking performance with the accuracy of one BLU, 20nm, can be obtained by using the MIC.
\end{abstract}

Key words: Linear-Motor-Driven Motion System, Macroscopic Model, Microscopic Model, Dual-Controller, Nanometer-Scale Trajectory.

\section{Introduction}

High precision motion control has become an essential requirement in today's advanced manufacturing systems and mechanical devices such as the machine tools, micro-manipulators, surface mounting robots, surgical tools, stepper and scanner. A dual servo system including a long-stroke stage (coarse stage) and a short-stroke stage (fine stage) is often used for achieving sub-micrometer accuracy over a range of a few millimeters movement ${ }^{(1)-(4)}$. A low-bandwidth long-stroke controller for a ball screw based servo motor or a voice coil motor (VCM) in large movement along with a high-bandwidth short-stroke controller for a piezoelectric actuator (PZT) in short moving-range of sub-micrometer accuracy are usually employed. The controllers of a dual servo system have to control two different types of actuators simultaneously. Pahk et al. ${ }^{(1)}$ developed an ultra precision positioning system with a servo-PZT actuator and a laser interferometer measurement system with $5 \mathrm{~nm}$ resolution. The authors proposed a dual servo loop controller and a Chebyshev filter to reduce the vibration of the motion stage. Tradition PID and PI controller were implemented in the dual servo controller. The experimental results indicated that it totally took 0.8 second to reach the $100 \mu \mathrm{m}$ target position with $10 \mathrm{~nm}$ positioning accuracy. A VCM-PZT actuator and a capacitance-type 
gap sensor with $10 \mathrm{~nm}$ resolution make the ultra precision system in Liu's work ${ }^{(2)}$. Liu et al. ${ }^{(2)}$ designed an integral variable structure controller (IVSC) to control the coarse stage and an impact force controller (IFC) to control the fine stage. It totally took 1.253 second to reach the target position of $450 \mu \mathrm{m}$ with $10 \mathrm{~nm}$ positioning accuracy. In both Pahk and Liu's works, the dual servo loop is changed from the long-stroke controller to the short-stroke controller as the table of long-stroke stage gets close to the target position about $10 \mu \mathrm{m}$. Schroeck et al. ${ }^{(3)}$ presented a PQ method to reduce a dual-input single-output (DISO) system into two standard controllers which are single-input single-output (SISO) systems. Choi et al. ${ }^{(4)}$ used the PQ method to design controller for PZT-VCM dual servo system with a sub-nanometer resolution capacitive position sensor. However, only simulation results were provided in Choi's work. The interaction between long-stroke actuator and the short-stroke actuator is the main disadvantage of the dual servo system. It is especially true when the motion is near the desired position; the long-stroke actuator tries to hold the table of long-stroke stage while the short-stroke actuator still forces the table of short-stroke stage moving to the target. The interaction force might lead the system to be unstable. The above dual servo system consisting of two controllers, two actuators, and two controlled plants is a kind of MIMO systems. Other than the MIMO system, the single-input single-output (SISO) system with single controller and single actuator has also been investigated for high precision control purpose ${ }^{(5)-(10)}$. Most investigations have been carried out with the friction compensation to achieve precise positioning. However, the bandwidth of the SISO system is similar to the low bandwidth of coarse stage with inevitable low-speed property in the dual servo system.

In recent years, linear-motor-driven motion systems have increasingly been applied to the higher performance CNC (computer numerical control) machine tools. For the linear-motor-driven motion system, the workpiece can be coupled rigidly to the motor. Linear-motor-driven motion system takes advantages over traditional ball-screw-driven system as there are no transmission errors such as backlash, lead screw error, belt stretch and gear tooth error nor a phenomenon where the load is hard to move small distances under low speed movement with high accuracy in the system. Only the nonlinear effects from the contact friction of the guideways will be involved. Several static and dynamic friction models such as Karnopp model ${ }^{(11)}$, Armstrong model ${ }^{(12)}$, Dahl model ${ }^{(13)}$ and Canudas model (LuGre model) ${ }^{(14)}$, have been proposed to design model-based friction compensators. Using accurate friction model to design controller in the presliding region will increase the positioning performance. Theoretically, both feedback-type friction compensator (FBFC) and feedforword-type friction compensator (FFFC) based on the LuGre friction model can be used for friction compensation. The FBFC uses the feedback velocity as input and generates the estimated friction into motion system for compensation; however, the FBFC based on the LuGre friction model may change the original well-designed dynamic system and the system may become unstable at times. On the contrary, the FFFC does not change the locations of original designed poles and the system response does not oscillate. However, model mismatches and incorrect friction parameters might lead to over compensation response in the presliding region ${ }^{(15)-(17)}$. For overcoming the disadvantage of the SISO system with single controller and single actuator, the idea of dual controllers, single actuator with dual controlled models is proposed to enhance positioning performance. Futami et al. ${ }^{(18)}$ proposed a dual controller for the identified three friction regions: 1) behavior of a linear spring, 2) behavior of a nonlinear spring, 3) behavior of normal sliding friction. The coarse controller, which consisted of position $\mathrm{P}$ and velocity feedback $\mathrm{P}$, was designed for region 3 , and the fine controller, which consisted of position integral controller, was designed for regions 1 and 2. For long-stroke movement, the table was controlled by the coarse controller. After the coarse position was completed, the coarse control force was released and rested for $50 \mathrm{~ms}$. Then, the fine 
controller took over the controller job. A resolution better than $1 \mathrm{~nm}$ was achieved with a capacitive gap sensor having resolution better than $1 \mathrm{~nm}$. The approach focused on the positioning accuracy without considering rapid transit.

In this paper, the mathematical model of the underlying system is separated into two parts, a microscopic model (within the presliding region) and a macroscopic model (beyond the presliding region). The microscopic model is a linearized model of the underlying system in the presliding region. As the system slides above the Stribeck velocity, only Coulomb friction exists and the macroscopic model is employed. Once system model parameters are identified in both regions, the corresponding MAC and MIC are designed individually; and further, a bumpless switching algorithm from the MAC to MIC is proposed. A linear-motor-driven motion system with $20 \mathrm{~nm}$ resolution of a fiber optic laser encoder is employed here for demonstrating the idea. In contrast with the dual servo system, the MAC and MIC serve as the long stroke (coarse stage) and short stroke (fine stage) controllers, respectively. The MAC can drive the table for large movement near to target and the MIC can shorten settling time with one basic length unit (BLU) accuracy (resolution of measurement instrument). Besides, the bandwidths of two closed-loop systems with MAC and MIC are about $10 \mathrm{~Hz}$ and $43 \mathrm{~Hz}$, respectively, which almost fit the requirement in the dual servo system. Compared with the position response with a single MAC, the proposed dual-controller has the advantages of faster response and higher precision in position.

The rest of this paper is organized as follows. The following section describes the hardware setup of our control system. Section 3 presents a macroscopic model of the linear-motor driven motion system with Coulomb friction model and a MAC design. Section 4 presents a linearized microscopic model of motion system and a MIC design. Section 5 presents a dual-controller with a switching algorithm for changing the controller from the MAC to MIC. Section 6 compares several experimental results for different controllers under microscopic movement. Section 7 draws conclusions.

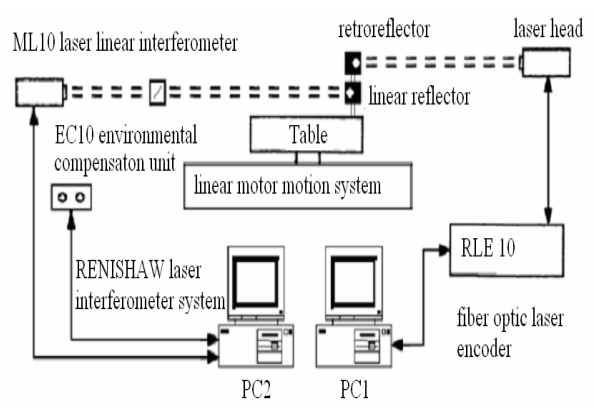

Fig.1 The experimental linear-motor-driven motion system together with the resolution calibration system.

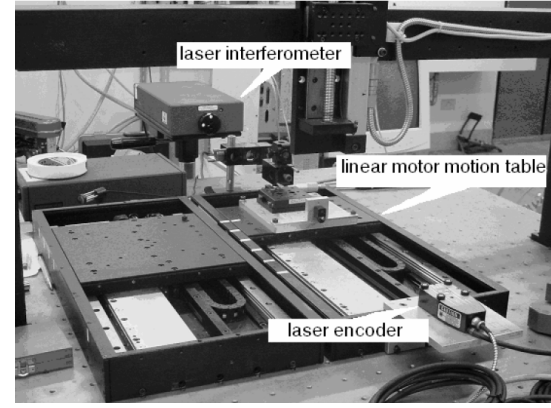

Fig. 2 A photo of the experimental system.

\section{Hardware Setup}

The experimental motion systems presented in Figs. 1 and 2 comprise the following components; a linear-motor-driven motion system, a laser displacement meter and a PC (PC1 in Fig. 1) with a DAC (digital to analog converter) and an encoder interface. The linear motor system has a linear motor (IL6-050A1) and an AC servo amplifier (SERVOSTAR CD) that operates in torque (current) mode, both of which are made by Kollmorgen Corporation ${ }^{(19)}$. Two sensors are used - one with a linear scale (RENISHAW RGH24Y, resolution 0.1 micrometer), providing position information for the current control of a servo amplifier, and a fiber optic laser encoder (RENISHAW RLE10) for measuring the displacement of the motion table with adjustable resolutions. The accuracy of the 
resolution supplied by RLE10 is influenced by such environmental factors as relative humidity, temperature, pressure and cosine errors. Therefore, calibration must be performed using another measurement instrument. For reducing the impact of the environmental factors, the RENISHOW laser interferometer system, which includes an environmental compensation unit (EC10) is used. Following calibration, the BLUs for coarse and fine resolutions are found to be $79.1 \mathrm{~nm}$ and $20.0 \mathrm{~nm}$, respectively. The choice of the resolution scale depends on the maximum encoder frequency (1 MHz in our system), the desired maximum velocity and the travel range. The velocity is estimated from the fiber optic laser encoder by $\alpha \beta$-filter ${ }^{(20)}$ in our motion system.

\section{Macroscopic Modeling and Controller Design}

\subsection{Macro-dynamic modeling of the mechanical system}

In general, the bandwidth of current loop is very fast as compared with mechanical system. If the high frequency modes are ignored, the system equation for the position of moving table can be simplified as

$$
J \ddot{x}+B \dot{x}+F_{1}=u
$$

where $J$ is the inertia (equivalent mass); $B \dot{x}+F_{1}$ is the friction force, and $u$ is the input force to the system generated by a current-controlled servo amplifier with a velocity loop controller and a position loop controller. The viscous coefficient, $B$, is regarded as a parameter of the controlled plant herein. Therefore, $F_{1}$ represents a friction force without a viscous friction term and it is generally a function of position, velocity and control input force.

\subsection{System parameter identification}

When the table slides above the Stribeck velocity, $v_{s}$, the friction force is reduced to the Coulomb friction, i.e., $F_{1}=F_{C}$. Only the inertia $J$, viscous coefficient $B$ and Coulomb friction $F_{C}$ have to be identified in the macroscopic model of the underlying system. In our previous work ${ }^{(21)}$, these three parameters can be identified by using disturbance observer (DOB) method and evaluated iteratively based on the orthogonal relations and trigonometric properties among the state variables. The identification procedures are briefly listed in the following.

Step 1: Perform a velocity tracking control experiment. Equation (2) gives a unidirectional reference command. Figure 3 presents the identification structure in which the PI controller must be tuned to uphold the assumptions of $v=v_{r}$ and $\dot{v}=\dot{v}_{r}$.

$$
v_{r}(t)=v_{0}+v_{1} \sin \left(\frac{2 \pi}{T_{p}} t\right)
$$

where $v_{0}>v_{1}>0$ to ensure that $v_{r}>0$ and $T_{p}$ is the period of this sinusoidal function. Notably $v_{r}$ must be larger than the Stribeck velocity, $v_{s}$, to guarantee the motion is operated beyond the presliding region.

Step 2: The control input and the corresponding velocity response data are fed into the DOB to yield a series of disturbance estimates $\hat{\tau}$. Calculate $\Delta J$ using the following equation.

$$
\Delta J=\frac{\sum_{N} \hat{\tau}[k] \dot{v}_{r}[k]}{\sum_{N} \dot{v}_{r}^{2}[k]}
$$

where $N$ denotes the sampling number over a period $T_{p}$.

Step 3: Update the estimate of inertia.

$$
\hat{J}_{\text {new }}=\hat{J}_{\text {old }}+\Delta J
$$


Step 4: Estimate the Coulomb friction $F_{C}$.

$$
\hat{F}_{C}=\frac{1}{N} \sum_{N} \hat{\tau}[k]
$$

Step 5: Calculate $\Delta B$ using the following equation.

$$
\Delta B=\frac{\sum_{N} \hat{\tau}[k] v_{r}[k]-\hat{F}_{C} \sum_{N} v_{r}[k]}{\sum_{N} v_{r}{ }^{2}[k]}
$$

Step 6: Update the viscous coefficient $B$.

$$
\hat{B}_{\text {new }}=\hat{B}_{\text {old }}+\Delta B
$$

Step 7: If $\Delta J$ and $\Delta B$ are not very small, then use the new parameters to redesign the DOB and go back to step 2 to evaluate new $J, B$ and $F_{C}$. If $\Delta J$ and $\Delta B$ are within preset tolerance, stop here.

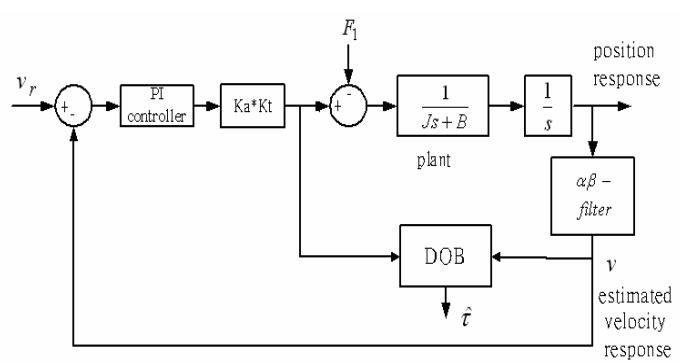

Fig. 3 A block diagram of the proposed identification experiment.
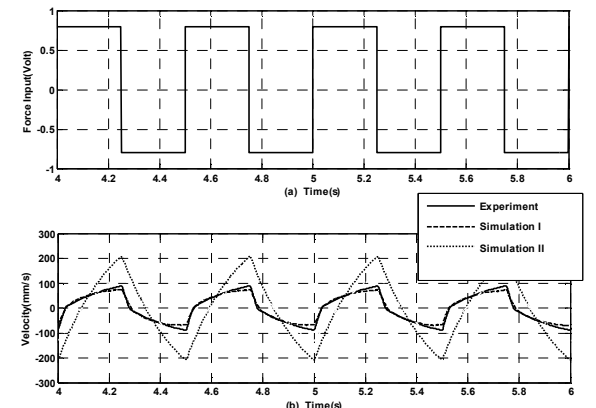

Fig. 4 (a) Force input and (b) velocity response of experimental and simulation results. The parameters of the simulation systems are listed in Table 2.

The estimated parameters are $J=4.46 \mathrm{~kg}, B=24.03 \mathrm{~kg} / \mathrm{s}$, and $F_{C}=4.29 \mathrm{~N}$ as listed in Table 1, in which other parameters for the experimental system are given for reference. Conventionally, a simple system without friction is used for modeling moving stage. The parameters $J$ and $B$ can be obtained by the step response method. For comparison, in our motion system a $1.5 \mathrm{~V}$ step force command is used and the identified parameters, $J=6.74 \mathrm{~kg}$ and $B=28.69 \mathrm{~kg} / \mathrm{s}$, are obtained. Table 2 presents two simulation cases with different models, identification methods and system parameters; and Figure 4 shows the simulation and experimental results. A 0.8 Volt step force command, presented in Fig. 4(a), is used to evaluate the time responses of two models. The solid Line in Fig. 4(b) plots the experimental results for the motion system herein. The results reveal that time response of the simulation I almost matches that of the real system, except when the command changes abruptly. Meanwhile, the simulated time response in simulation II has a larger magnitude than the real response. Thus, the MAC is designed based the parameters listed in Table 1.

Table 1 Parameters of the experimental system.

\begin{tabular}{||l||l|l||}
\hline Symbol and name & Value & Unit \\
\hline \hline$J$, inertia & 4.46 & $\mathrm{~kg}$ \\
\hline$F_{C}$, viscous friction coefficient & 24.03 & $\mathrm{~kg} / \mathrm{s}$ \\
\hline$K_{t}$, force constant of motor & 28.5 & $\mathrm{~N} / \mathrm{A}$ \\
\hline$K_{a}$, gain of current driver & 0.349 & $\mathrm{~A} / \mathrm{V}$ \\
\hline$T$, sampling rate & 0.0005 & $\mathrm{~s}$ \\
\hline$D$, pole-pitch of motor & 32 & $\mathrm{~mm}$ \\
\hline$v_{S}$, Stribeck velocity & 4.4148 & $\mathrm{~mm} / \mathrm{s}$ \\
\hline Breakaway point & 10 & $\mu \mathrm{m}$ \\
\hline
\end{tabular}

Table 2 Properties for each simulation system.

\begin{tabular}{||l|l|l|l||}
\hline & $\begin{array}{l}\text { System } \\
\text { model }\end{array}$ & $\begin{array}{l}\text { Identification } \\
\text { method }\end{array}$ & $\begin{array}{l}\text { Identified } \\
\text { Parameters }\end{array}$ \\
\hline \hline $\begin{array}{l}\text { Simulation I } \\
\text { (dash-line) }\end{array}$ & $\begin{array}{l}\text { Containing } \\
F_{C}, \text { and }\end{array}$ & $\begin{array}{l}\text { Method in } \\
\text { Ref. [20] }\end{array}$ & $\begin{array}{l}J=4.46 \mathrm{~kg}, \\
B=24.03 \mathrm{~kg} / \mathrm{s}, \\
F_{C}=4.29 \mathrm{~N}\end{array}$ \\
\hline $\begin{array}{l}\text { Simulation II } \\
\text { (dotted-line) }\end{array}$ & $\begin{array}{l}\text { Only } \\
\text { containing }\end{array}$ & $\begin{array}{l}\text { Step response } \\
\text { method }\end{array}$ & $\begin{array}{l}J=6.74 \mathrm{~kg} \\
B=28.69 \mathrm{~kg} / \mathrm{s}\end{array}$ \\
\hline
\end{tabular}




\subsection{MAC design}

Figures 5 and 6 present the block diagrams of two MAC structures. The MAC I consists of a position P controller $\left(C_{p}\right)$, a velocity feedforward controller $\left(F_{v}\right)$, a velocity PI controller $\left(C_{v}\right)$ and an acceleration feedforward controller $\left(F_{a}\right)^{(22)}$; the MAC II includes the MAC I, a friction compensator (FC) and a disturbance observer (DOB) which is often employed for high-accuracy position control. The transfer functions of the controllers in MAC I are as follows:

$$
\begin{aligned}
& C_{p}(s)=K_{p p} \\
& F_{v}(s)=s \\
& C_{v}(s)=K_{v p}+\frac{K_{v i}}{s} \\
& F_{a}(s)=\frac{J_{n} s+B_{n}}{K}
\end{aligned}
$$

where $K_{p p}$ denotes the proportional control gain in position controller; $K_{v p}$ and $K_{v i}$ are the proportional and integral gains, respectively, in velocity controller; $J_{n}$ and $B_{n}$ are the nominal parameters of the plant; and $K=K_{a} K_{t}$ is the motor constant. Notably, the differentiation in Eq. (9) and (11) are implemented by the $\alpha \beta$-filter ${ }^{(20)}$. Based on the Coulomb friction model, the FC is designed for improving the tracking performance. The residual effect and the uncertainties of plant parameters are reduced by the disturbance observer $(\mathrm{DOB})^{(23,24)}$. Since the DOB loop is an inner loop with respect to the velocity loop, the design becomes independent of velocity and position loops. Figure 7 draws the block diagram of the DOB, where $P_{n}(s)$ is the nominal plant; $Q(s)$ is a low-pass filter to make sure that $Q(s) P_{n}^{-1}(s)$ is proper function and can be realized. The bandwidth of $Q(s)$ for control purpose is chosen to let the low-frequency dynamics of $Q(s)$ be close to 1 for disturbance and model uncertainties rejection. The high-frequency dynamics must be close to 0 for sensor noise rejection. Each controller gains are designed for the underlying linear-motor-system with $10 \mathrm{~Hz}$ position $\mathrm{P}$ controller bandwidth, $60 \mathrm{~Hz}$ velocity PI-controller bandwidth and $10 \mathrm{~Hz}$ DOB low-pass bandwidth. The controller gains are listed in Table 3

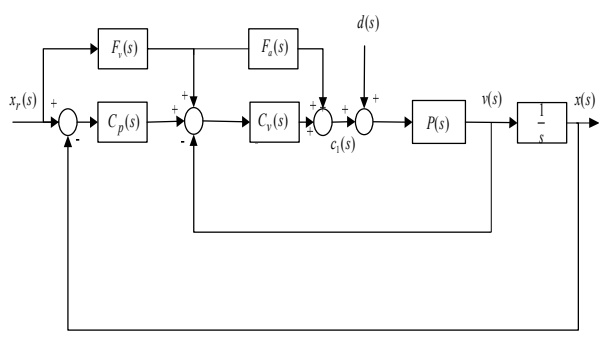

Fig. 5 A block diagram of the MAC I.

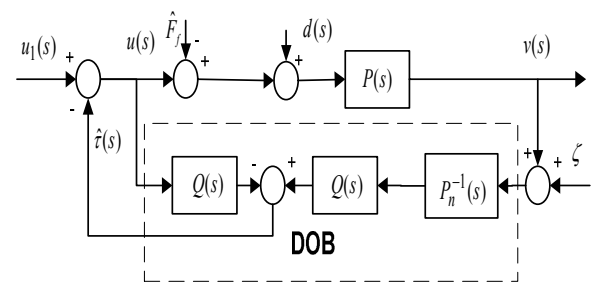

Fig. 7 Block diagram of the disturbance observer for compensation.

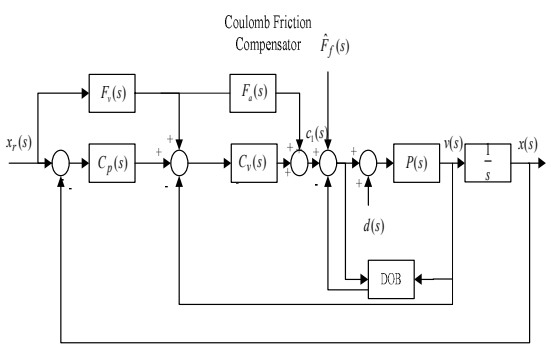

Fig. 6 A block diagram of the MAC II.

Table 3 The values of the plant parameters and controller gains.

\begin{tabular}{||l|l|l||}
\hline Symbol and name & Value & Unit \\
\hline \hline$J_{n}$, nominal value of $J$ & 4.46 & $\mathrm{~kg}$ \\
\hline$B_{n}$, nominal value of $B$ & 24.03 & $\mathrm{~kg} / \mathrm{s}$ \\
\hline$K_{p p}$, P-gain in position controller & 62.83 & $1 / \mathrm{s}$ \\
\hline$K_{v p}$, P-gain in velocity controller & 335.67 & $\mathrm{~V} / \mathrm{s}$ \\
\hline$K_{v i}$, I-gain in velocity controller & 63767.45 & $\mathrm{~V}$ \\
\hline$K, \quad$ motor constant & 9.947 & $\mathrm{~N} / \mathrm{V}$ \\
\hline
\end{tabular}




\section{Microscopic Modeling and Controller Design}

The interface between two contact rigid bodies can be modeled as numerous elastic bristles. If the motion is operated in the presliding region, the movement of the linear-motor-driven motion system is almost the same as the mean bristle deflection between two contact rigid bodies. A linearized model to describe the overall system including the nonlinear friction model is employed here.

\subsection{Identification procedures}

The identification procedures for the microscopic motion system are described below:

Step 1: Choose a Pseudo random binary sequence (PRBS) signal ${ }^{(25)}$ :

The magnitude of the PRBS signal is chosen carefully for preventing the motion system from leaving the presliding region (The breakaway point is around $10 \mu \mathrm{m}$ in the underlying system). The PRBS signal is produced from the function IDINPUT in Matlab with length $M=5000$ and shift frequency $400 \mathrm{~Hz}$.

Step 2: Obtain the position response of the microscopic motion system:

The PRBS signal is the input force into the motor driver from the 14-bit D/A card in PC. The position of the motor is obtained from a fiber optic laser encoder (RENISHAW RLE10) with 20nm resolution.

Figure 8 represents the PRBS input signal and the position response. All the position data points are within $6 \mu \mathrm{m}$. This implies that the bristle of the motion stage is not broken and the motion is in the presliding region.

Step 3: Perform Model identification:

The PRBS input voltage and position response in step 2 are employed to identify our motion system by using the System Identification toolbox in Matlab. The N4SID (N' Subspace State Space System ID) algorithm is adopted to estimate the model of the microscopic motion system. The order of the estimated model is chosen according to the model fitness index of the estimated model. The estimated model of the $8^{\text {th }}$ order continuous system with the highest model fitness index 87.7 is obtained as

$$
G_{m}(\mathrm{~s})=\frac{-282.169(\mathrm{~s}-3269)(\mathrm{s}+889.6)(\mathrm{s}+48.39)\left(\mathrm{s}^{2}+71.15 \mathrm{~s}+2892000\right)\left(\mathrm{s}^{2}+64.23 \mathrm{~s}+6346000\right)}{(\mathrm{s}+216.5)(\mathrm{s}+19.1)\left(\mathrm{s}^{2}+633.7 \mathrm{~s}+525000\right)\left(\mathrm{s}^{2}+124.3 \mathrm{~s}+2906000\right)\left(\mathrm{s}^{2}+55.72 \mathrm{~s}+6317000\right)}
$$

The frequency response of the $8^{\text {th }}$ order continuous system with eight poles and seven zeros is shown in dashed-line in Fig. 9. There exists a non-minimum phase zero at 3269 which is far away from imaginary axis of the s plane. The non-minimum phase zero can be treated as an infinite zero and thus omitted.

Step 4: Perform Model reduction:

From the estimation results in step 3, an $8^{\text {th }}$ order continuous model is obtained. In order to simplify the controller design, model reduction is performed in this step. The model fitness index is 82.52 if the model order is chosen as three. The estimated system of the $3^{\text {rd }}$ order continuous system is obtained as

$$
G_{m}(s)=\frac{-965.1033(\mathrm{~s}-1836)(\mathrm{s}+134.2)}{(\mathrm{s}+37.76)\left(\mathrm{s}^{2}+758.5 \mathrm{~s}+343100\right)} \quad[\mu \mathrm{m} / \mathrm{V}]
$$

The model has three poles and two zeros. A non-minimum phase zero, 1836, exists and can be treated as an infinite zero. With the omission of the non-minimum phase zero, match the DC-gain of the transfer function to that of the $8^{\text {th }}$ order transfer function in Eq. (12). The new $3^{\text {rd }}$ order transfer function of the estimated model is

$$
G_{m}(s)=\frac{1771929.6128(\mathrm{~s}+134.2)}{(\mathrm{s}+37.76)\left(\mathrm{s}^{2}+758.5 \mathrm{~s}+343100\right)} \quad[\mu \mathrm{m} / \mathrm{V}]
$$

The frequency response of the $3^{\text {rd }}$ order system neglecting the non-minimum phase zero is shown in solid-line in Fig. 9 and it almost fits the response of the $8^{\text {th }}$ order system under $100 \mathrm{~Hz}$. 

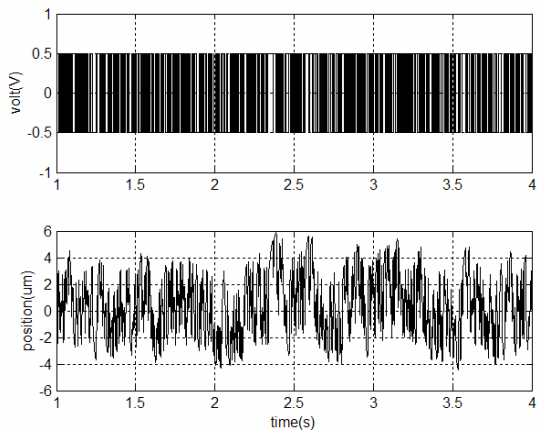

Fig. 8 The PRBS input signal and position response.

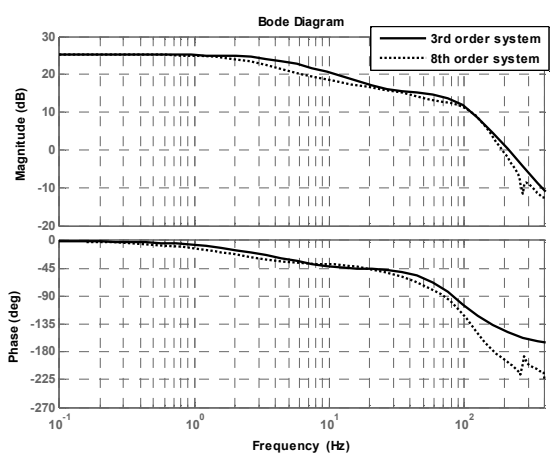

Fig. 9 Frequency responses of the $8^{\text {th }}$ order system and the reduced order system.

\subsection{The MIC design}

The objective of the MIC is to achieve perfect tracking performance with one BLU, 20nm accuracy. A simple but effective position PI controller based on the $3^{\text {rd }}$ order estimated model, Eq. (14), is designed. Table 4 lists five sets of controller gains designed by pole placement method and also shows the bandwidth, the poles, and the zeros for each closed-loop system. Two sinusoidal position commands are fed to the servo loop to evaluate the performance for each system. For preventing the oscillation effect from the quantization effect induced by the encoder with $20 \mathrm{~nm}$ resolution, the quantized sinusoidal commands are designed as follows and also shown in Fig. 10(a) and Fig. 11(a).

$$
\begin{aligned}
& x_{r}(t)=\operatorname{int}\left(\frac{0.2 \cdot(1-\cos (2 \pi \cdot 0.1 \cdot t))}{0.02}+0.5\right) \times 0.02 \mu \mathrm{m} \\
& x_{r}(t)=\operatorname{int}\left(\frac{0.2 \cdot(1-\cos (2 \pi \cdot 1 \cdot t))}{0.02}+0.5\right) \times 0.02 \mu \mathrm{m}
\end{aligned}
$$

where int $(\mathrm{A})$ rounds the number $\mathrm{A}$ to the nearest integer.

The performance comparisons among the five closed loop systems are performed according to two performance indexes. The first index indicating the maximum tracking error is defined as

$$
E_{\max }=\max _{0 \leq t \leq 12.0}\left|x_{r}-x\right|
$$

The second index indicating the root-mean-square tracking error is defined as

$$
E_{r m s}=\sqrt{\frac{1}{N} \sum_{N}\left(x_{r}-x\right)^{2}} \quad, 0 \leq t \leq 12.0
$$

where $N$ denotes the sampling number and $x$ is the position response.

Table 4 Five sets of controller gains and the closed-loop system properties.

\begin{tabular}{||c||c||c||c||c||c|}
\hline \hline Controller & Case I & Case II & Case III & Case IV & Case V \\
\hline \hline$k_{p}$ & 0.0373 & 0.0373 & 0.1242 & 0.12 & 0.1242 \\
\hline \hline$k_{i}$ & 5 & 24.0469 & 31.6863 & 48 & 80.0705 \\
\hline \hline Bandwidth & $10 \mathrm{~Hz}$ & $35 \mathrm{~Hz}$ & $43 \mathrm{~Hz}$ & $90 \mathrm{~Hz}$ & $117 \mathrm{~Hz}$ \\
\hline \hline Poles & $-353.1 \pm 609.7 \mathrm{i}$ & $-323.3 \pm 460.4 \mathrm{i}$ & $-309.1 \pm 584.4 \mathrm{i}$ & $-275.7 \pm 571.5 \mathrm{i}$ & $-198.2 \pm 564.2 \mathrm{i}$ \\
& $-45.02 \pm 6.67 \mathrm{i}$ & $-74.85 \pm 100.1 \mathrm{i}$ & $-89.1 \pm 83.6 \mathrm{i}$ & $-122.4 \pm 97.7 \mathrm{i}$ & $-199.9 \pm 78.0 \mathrm{i}$ \\
\hline \hline Zeros & -40.26 & -644.7 & -255.1 & -386.5 & -644.7 \\
& -134.2 & -134.2 & -134.2 & -134.2 & -134.2 \\
\hline \hline
\end{tabular}



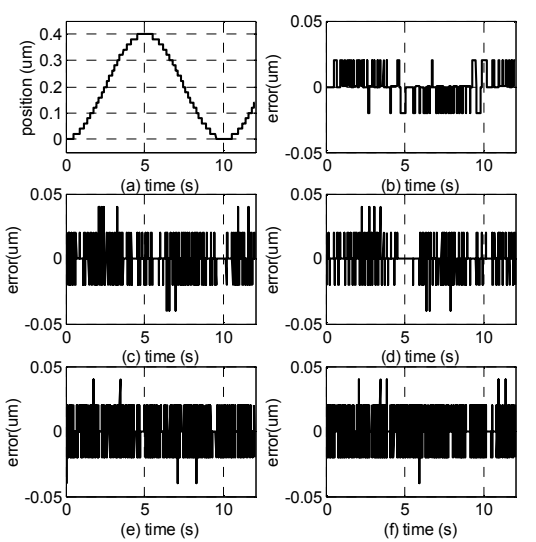

Fig. 10 (a) The $0.1 \mathrm{~Hz}$ quantized position command and position error for five cases:

(b) Case I; (c) Case II; (d) Case III;

(e) Case IV; (f) Case V.

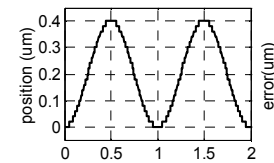

(a) time (s)
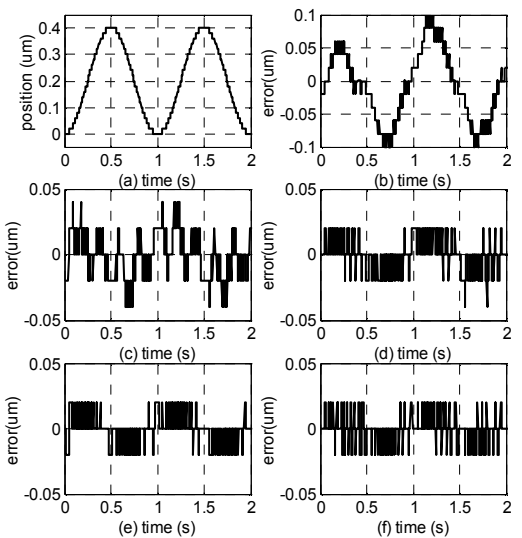

Fig. 11 (a) The $1 \mathrm{~Hz}$ quantized position command and position error for five cases: (b) Case I; (c) Case II; (d) Case III; (e) Case IV; (f) Case V.

Figure 10 presents the experimental results for the $0.1 \mathrm{~Hz}$ quantized command. All the maximum tracking error is about one BLU, i.e. $20 \mathrm{~nm}$, and all the RMS errors are smaller than $12 \mathrm{~nm}$. Notably, there appear sufficient chattering phenomenon in Case VI and Case $\mathrm{V}$. The quantized command acts as a micro-step command which induces high frequency response in Case IV and Case V. Thus, the Case I, II and III controller gains are suitable for this condition. As for the second position command, the experimental results of the $1 \mathrm{~Hz}$ quantized command are shown in Fig. 11. The tracking errors of the Case III, Case IV, and Case V are with $1 \mathrm{BLU}, 20 \mathrm{~nm}$. In the meantime, the RMS error of Case III, Case IV, and Case V are smaller than $12 \mathrm{~nm}$. Thus, the controller gains in Case III, Case IV, and Case V, are suitable to control the microscopic trajectories near $1 \mathrm{~Hz}$ frequency. In summary, the Case III is chosen as the MIC gains in the following experiments.

\section{Dual Controller Design}

The MAC II is adopted in the proposed dual-controller, while MAC I is used in the experiments for comparison. As motion starts, the MAC II is actuated to move the motion stage near the desired position. When the motion stage moves near the desired position, control action is shifted to the MIC to shorten the positioning time. Figure 12 shows the block diagram of a switching algorithm from the MAC II control force, $c_{1}$, to the MIC control force, $c_{2}$. In general, the control force of the MIC is not equal to that of the MAC II at switching instant. The discontinuity between two controller forces will induce the overshoot phenomenon during switching. In order to eliminate this phenomenon, the initial condition of the MIC should be adjusted properly. Since the MIC is a PI controller, the controlled force $c_{2}$ can be computed as

$$
c_{2}=k_{p} \cdot e_{x}+k_{i} \cdot \int e_{x}=k_{p} \cdot e_{x}+k_{i} \cdot \operatorname{sum}\left(e_{x}\right),
$$

where $e_{x}$ is the position error, $\operatorname{sum}\left(e_{x}\right)$ the integration of the position error, and $k_{p}, k_{i}$ the PI controller gains of the MIC. At the switching moment, let the actuating forces between the MAC II and the MIC be the same, i.e.,

$$
c_{1}\left(t_{\text {switch }}\right)=c_{2}\left(t_{\text {switch }}\right) \text {. }
$$

where the controlled force $c_{2}$ is obtained from the MAC II. Therefore, the initial condition of the integration position error of the MIC is

$$
\operatorname{sum}\left(e_{x}\right)=\left(c_{1}\left(t_{\text {switch }}\right)-k_{p} \cdot e_{x}\right) / k_{i},
$$

As for the switching condition, it is chosen according to the position response around the presliding range. It can be described as 


$$
u_{1}= \begin{cases}c_{1} & ,|A-x|>\Delta x \\ c_{2} & ,|A-x| \leq \Delta x\end{cases}
$$

where $x$ is the position response, $A$ is the desired position and $\Delta x$ is the switching distance.

\section{Experimental results}

A $1 \mathrm{~mm}$ positioning command is applied to the motion stage to evaluate the positioning performance under various switching conditions.

Fig. 13(a) illustrates the command which has the following form.

$$
x_{r}(t)= \begin{cases}A\left[6\left(\frac{t}{T_{a c c}}\right)^{5}-15\left(\frac{t}{T_{a c c}}\right)^{4}+10\left(\frac{t}{T_{a c c}}\right)^{3}\right] & , 0 \leq t \leq T_{a c c} \\ A & , t>T_{a c c}\end{cases}
$$

where $A=1(\mathrm{~mm})$ and $T_{a c c}=2.5(s)$.

Three performance indexes are adopted, or

$$
\begin{aligned}
& E_{\text {overshoot }}=\max _{x \geq A}|A-x| \\
& E_{\text {rms }}^{\prime}=\sqrt{\frac{1}{N} \sum_{N}(A-x)^{2}} \quad, x>A, 2.0 \mathrm{~s} \leq \mathrm{t} \leq 3.5 \mathrm{~s} .
\end{aligned}
$$

$T_{s}$ : Settling time

where $E_{\text {overshoot }}$ is the maximum overshoot of the response curve over the target $A ; E_{r m s}^{\prime}$ indicates the root-mean-square tracking error after the switching algorithm is triggered, and the settling time $T_{s}$ is defined as the time for the position response from motion start to reach and stay within $\pm 20 \mathrm{~nm}$ from the target position. The switching algorithm is triggered when the position response approaches to the desired position within certain distance $\Delta x$. Five switching distances, $0.5 \mu \mathrm{m}, 1.0 \mu \mathrm{m}, 5.0 \mu \mathrm{m}, 10.0 \mu \mathrm{m}$ and $13.0 \mu \mathrm{m}$, are adopted to evaluate the switching performance. Figures 13 (b) (f) illustrate the position errors of five cases. Three performance indexes for five cases are listed in Table 5 where the settling time $T_{S}$ is below $2.67 \mathrm{sec}$ for all cases. In particular, the case of switching distance $10.0 \mu \mathrm{m}$ is the best one, which is consistent with the breakaway point. If the switching distance is smaller than $10.0 \mu \mathrm{m}$, larger values of $E_{\text {overshoot }}$ and $E_{r m s}^{\prime}$ will be observed due to the model mismatch in the presliding region. For comparison, the MAC I and MAC II without switching algorithm are applied. Figures 14 (a) and (b) illustrate the position errors for the MAC I and MAC II, respectively and the performance indexes are also listed in Table 5. In the macroscopic region, the performance of MAC II is better than that of MAC I; however, MAC II with disturbance observer based controller might generate limit cycles which severely decreases positioning accuracy as the system reaches steady-state positioning ${ }^{(26)}$. The experimental results reveal that the positioning performance using any MAC is inferior to that using dual-controller. In summery, the application of dual-controller with switching distance $10 \mu \mathrm{m}$ is the best and it takes 2.59 second for the motion table to reach the target position of $1000 \mu \mathrm{m}$ with the positioning accuracy of one BLU, 20nm, over $29 \%$ improvement in $T_{s}, 91 \%$ improvement in $E_{r m s}^{\prime}$ and $93 \%$ improvement in $E_{\text {overshoot }}$ when compared with the results using any single MAC.

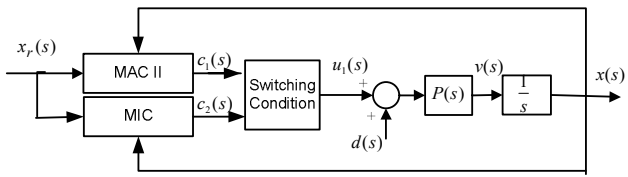

Fig. 12 A control structure showing dual-controller with switching algorithm. 
Table 5 The performance indexes for each controller.

\begin{tabular}{||c||c||c||c|c||}
\hline Controllers & $\begin{array}{c}\text { Switching } \\
\text { distance }\end{array}$ & $\begin{array}{c}E_{r m s}^{\prime}(\mu \mathrm{m}) \\
x>A \text { and } \\
2.0 \mathrm{~s} \leq t \leq 3.5 s\end{array}$ & $\begin{array}{c}E_{\text {overshoot }}(\mu \mathrm{m}) \\
x>A\end{array}$ & $T_{S}(\mathrm{~s})$ \\
\hline \hline Dual-controller & $0.5 \mu \mathrm{m}$ & 0.8346 & 0.14 & 2.602 \\
\hline \hline Dual-controller & $1.0 \mu \mathrm{m}$ & 1.2021 & 0.16 & 2.642 \\
\hline \hline Dual-controller & $5.0 \mu \mathrm{m}$ & 0.8347 & 0.1 & 2.670 \\
\hline \hline Dual-controller & $10.0 \mu \mathrm{m}$ & 0.5711 & 0.08 & 2.590 \\
\hline Dual-controller & $13.0 \mu \mathrm{m}$ & 0.6370 & 0.08 & 2.608 \\
\hline \hline MAC I & None & 12.2788 & 1.08 & 3.640 \\
\hline \hline MAC II & None & 6.1025 & 0.34 & $>>5.0$ \\
\hline
\end{tabular}

Table 6 The performance indexes under nanometer-scale triangular trajectory.

\begin{tabular}{||c||c|c||}
\hline Controllers & $E_{r m s}(\mu \mathrm{m})$ & $E_{\max }(\mu \mathrm{m})$ \\
\hline \hline MIC & $1.11 \times 10^{-2}$ & $2.00 \times 10^{-2}$ \\
\hline \hline MAC I & $2.36 \times 10^{-2}$ & $6.00 \times 10^{-2}$ \\
\hline \hline MAC II & $2.37 \times 10^{-2}$ & $6.00 \times 10^{-2}$ \\
\hline
\end{tabular}

Table 7 The performance indexes under nanometer-scale sinusoidal trajectory.

\begin{tabular}{||l||l|l||}
\hline Controllers & $E_{r m s}(\mu \mathrm{m})$ & $E_{\max }(\mu \mathrm{m})$ \\
\hline \hline MIC & $1.15 \times 10^{-2}$ & $2.00 \times 10^{-2}$ \\
\hline \hline MAC I & $7.16 \times 10^{-2}$ & $16.00 \times 10^{-2}$ \\
\hline \hline MAC II & $2.15 \times 10^{-2}$ & $6.00 \times 10^{-2}$ \\
\hline
\end{tabular}
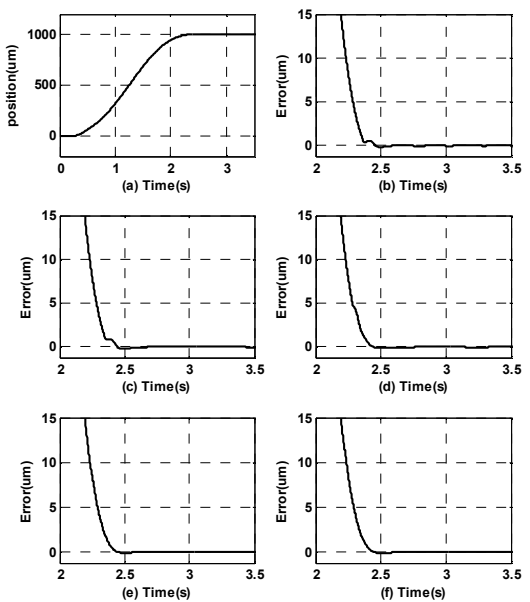

Fig. 13 (a) The $1 \mathrm{~mm}$ position command and position errors after $2 \mathrm{~s}$ for various $\Delta x$ :

(b) $0.5 \mu \mathrm{m}$, (c) $1.0 \mu \mathrm{m}$, (d) $5.0 \mu \mathrm{m}$,

(e) $10.0 \mu \mathrm{m}$, and (f) $13.0 \mu \mathrm{m}$.
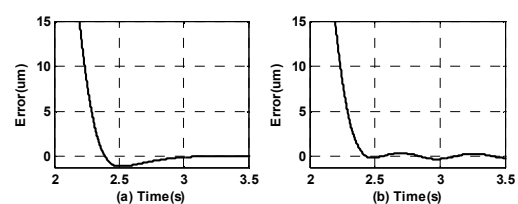

Fig. 14 The position errors of

(a) MAC I, and (b) MAC II.

Next, experiments of two nanometer-scale trajectories are conducted; one is a nanometer-scale triangular trajectory and the other is a nanometer-scale $1 \mathrm{~Hz}$ sinusoidal trajectory. The MIC and above two MACs are applied to evaluate the tracking performance. Figure 15 and Figure 16 display the position command, position responses and the tracking errors of three controllers for the nanometer-scale triangular trajectory and the nanometer-scale $1 \mathrm{~Hz}$ sinusoidal trajectory, respectively. Table 6 and Table 7 list their corresponding performance indexes, $E_{\max }$ and $E_{r m s}$. The experiments reveal that the MIC is superior to any MAC and it can control the linear-motor-driven motion system with one BLU tracking error under the nanometer-scale trajectories. 

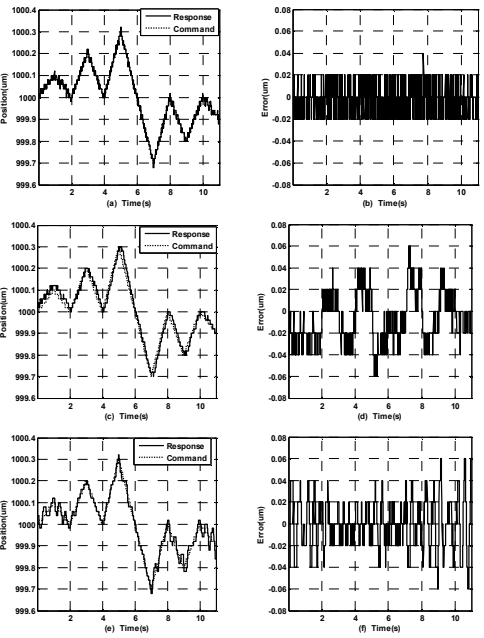

Fig. 15 The position responses under a nanometer-scale triangular trajectory, (a) position response and

(b) position error for the MIC;

(c) position response and

(d) position error for the MAC I;

(e) position response and

(f) position error for the MAC II
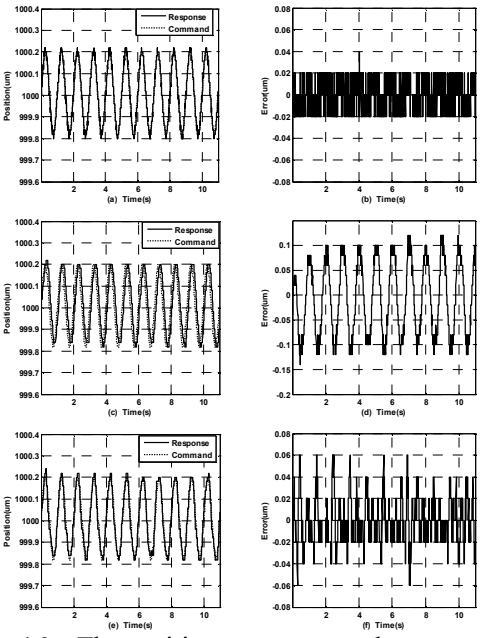

Fig. 16 The position responses under a nanometer-scale sinusoidal trajectory, (a) position response and

(b) position error for the MIC;

(c) position response and

(d) position error for the MAC I;

(e) position response and

(f) position error for the MAC II.

\section{Conclusion}

Instead of using a dual servo system including a long-stroke stage (coarse stage) and a short-stroke stage (fine stage) for achieving sub-micrometer accuracy over a range of a few millimeters movement, the idea of dual controllers, single actuator with dual controlled models was proposed in this paper. Following this idea, the authors presented the design procedures of a dual-controller with switching algorithm. A linear-motor-driven motion system with $20 \mathrm{~nm}$ resolution of a fiber optic laser encoder was employed here to demonstrate the idea. In contrast with the dual servo system, the MAC and the MIC function as the long stroke (coarse stage with low bandwidth) and short stroke (fine stage with high bandwidth) controllers, respectively. The MAC can drive the system for large movement near to target and the MIC can shorten settling time with the accuracy of one BLU. The experimental results have demonstrated the effectiveness of the proposed method.

\section{Acknowledgements}

The authors would like to thank the National Science Council of the Republic of China for financially supporting this paper under contract No. NSC93-2212-E-009-006

\section{References}

(1) Pahk, H.J., Lee, D.S., and Park, J.H., Ultra precision positioning system for servo motor-piezo actuator using the dual servo loop and digital filter implementation, Int. Jour. of Machine Tools and Manufacture, Vol. 41 (2001), pp. 51-63.

(2) Liu, Y.T, Fung, R.F., and Wang, C.C., Precision position control using combined piezo-VCM actuators, Precision Engineering, Vol. 29 (2005), pp.411-422.

(3) Schroeck, S.J., Messner, W.C., and McNab, R.J., On compensator design for linear time-invariant dual-input single-output systems, IEEE/ASME Tran. on Mechatronics, Vol. 6, No. 1 (2001), pp. 50-57.

(4) Choi, H.S., Song, C.W., Han, C.S., Choi, T.H., Lee, N.K., Lee, H.W., and Na, K.H., Designing compensator of dual servo system for high precision position control, SICE Annual Conf. 2003, pp.2732-2737.

(5) Awabdy, B.A., Shih, W., and Auslander, D. M. Nanometer positioning of a linear motion stage under static loads, IEEE/ASME Trans. on Mechatronics, Vol. 3, No. 2 (1998), pp. 113-119. 
(6) Iwasaki, M., Shibata, T., Matsui, N., Disturbance-observer-based nonlinear friction compensation in table drive system, IEEE/ASME Trans. on Mechatronics, Vol. 4, No. 1 (1999), pp. 3-8.

(7) Lee, H.S., Tomizuka, M., Robust motion controller design for high-accuracy positioning systems, IEEE Trans. on Industrial Electronics., Vol. 43, No. 1 (1996), pp. 48-55.

(8) Park, E.C., Lim, H., and Choi, C.H., Position control of X-Y table at velocity reversal using presliding friction characteristics, IEEE trans. on Control Systems Technology, Vol.11, No.1 (2003), pp. 24-31.

(9) Ray, L.R., Ramasubramanian, A., and Townsend, J., Adaptive friction compensation using extended Kalman-Bucy filter friction estimation, Control Engineering Practice, Vol. 9 (2001), pp. 169-179.

(10) Tan, K.K, Lee, T.H., Huang, S.N., and Jiang, X., Friction modeling and adaptive compensation using a relay feedback approach, IEEE Trans. on Industrial Electronics, Vol. 48 , No. 1 (2001), pp. 169-176.

(11) Karnopp, D., Computer simulation of stick-slip friction in mechanical dynamic systems, ASME J. of Dynamic systems, Measurement and Control, Vol. 107, No. 1 (1985), pp. 100-103.

(12) Armstrong-Helouvry, B., Control of machines with friction, (1991), Kluwer Academic Publishers, Nowell, MA.

(13) Dahl, P., Solid friction damping of mechanical vibrations, AIAA Journal, Vol. 14, No. 12 (1976), pp. 1675-1682.

(14) Canudas de Wit, C., Olsson, H., Astrom, K.J., and Lischinsky, P., A new model for control of systems with friction, IEEE Trans. on Automatic Control, Vol. 40, No. 3 (1995), pp. 419-425.

(15) Swevers, J., Al-Bender, F., Ganseman, C.G., and Prajogo, T., An integrated friction model structure with improved presliding behavior for accurate friction compensation, IEEE Trans. on Automatic Control, Vol. 45, No. 4 (2000), pp. 675-686.

(16) Lee, H.S., Tomizuka, M., Robust motion controller design for high-accuracy positioning systems, IEEE Trans. on Industrial Electronics, Vol. 43, No. 1 (1996), pp. 48-55.

(17) Tsai, M.C., Chiu, I.F., and Cheng, M.Y., Design and implementation of command and friction feedforward control for CNC motion controllers, IEE Proc. of Control Theory and Applications, Vol. 151, No. 1 (2004), pp. 13-20.

(18) Futami, S., Furutani, A., and Yoshida, S., Nanometer positioning and its micro-dynamics, Nanotechnology, Vol. 1, No. 1 (1990), pp. 31-37.

(19) PLATINUM DDL and SERVOSTAR setup Guide, (2002-6), Document number: M-LN-016-0702, Kollmorgen, a Danaher Motion Company.

(20) Edward P.C., Digital filtering, Chap. 9 (1992), Houghton Mifflin Co..

(21) Shih, Y.T., The High Precision Control for a Linear-Motor-Driven Motion Stage with Friction Compensation, (2004), Doctor Thesis, National Chiao-Tung University, Hsinchu City, Taiwan.

(22) Chen C. S. and Lee, A. C., New Direct Velocity and Acceleration Feedforward Tracking Control in a Retrofitted Milling Machine, Int. J. of Japan Soc. Prec. Eng., Vol. 33, No. 3, (1999), pp. 178-184.

(23) Umeno T. and Hori Y., Robust Speed Control of DC Servomoters Using Modern Two Degree-of-Freedom Controller Design, IEEE Trans. on Industrial Electronics, Vol.38, No.5 (1991-10), pp. 363 368.

(24) Umeno T., Kaneko T. and Hori, Y., Robust Servosystem Design with Two Degrees of Freedom and its Application to Novel Motion Control of Robot Manipulators, IEEE Trans. on Industrial Electronics, Vol.40, No.5 (1993-10), pp. 473-485.

(25) Söderström, T., and Stoica, P, Syetem Identification, (1989), Prentice Hall, UK, pp.137-145.

(26) Horng R.H., Chou H.L. and Lee, A.C., Rejection of Limit Cycles Induced from Disturbance Observers in Motion Control, IEEE Trans. on Industrial Electronics, Vol. 53, No. 6 (2006), pp. 1770-1780. 